\title{
Analysis of Data from Surface Detector Stations of the AugerPrime Upgrade
}

\author{
Álvaro Taboada ${ }^{* a, b}$ for the Pierre Auger Collaboration ${ }^{\dagger c}$ \\ ${ }^{a}$ Karlsruher Institut für Technologie (KIT), Institut für Kernphysik (IKP), Karlsruhe, Germany \\ ${ }^{b}$ Instituto de Tecnologías en Detección y Astropartículas (ITeDA), Buenos Aires, Argentina \\ ${ }^{c}$ Observatorio Pierre Auger, Av. San Martín Norte 304, 5613 Malargüe, Argentina \\ E-mail: auger_spokespersons@fnal.gov \\ Full author list: http://www.auger.org/archive/authors_icrc_2019.html
}

\begin{abstract}
Measuring the different components of extensive air showers is of key importance in reconstructing the mass composition of ultra-high energy cosmic rays. AugerPrime, the upgrade of the Pierre Auger Observatory, aims to enhance the sensitivity of its surface detector to the masses of cosmic rays by installing a $3.8 \mathrm{~m}^{2}$ plastic scintillator detector on top of each of the 1660 Water-Cherenkov Detectors (WCDs). This Scintillator Surface Detector (SSD) provides a complementary measurement which allows for disentanglement of the electromagnetic and muonic shower components. Another important improvement of AugerPrime are the surface-detector electronics. The new electronics will process signals from the WCD and the SSD with higher sampling frequency and enhanced resolution in signal amplitude. Furthermore, a smaller photomultiplier tube will be added to each WCD, thus increasing its dynamic range. Twelve upgraded surface detector stations have been operating since September 2016. Additionally, seventy-seven SSDs have been deployed and are taking data since March 2019. In this work, the analysis of the data from these detectors is presented.
\end{abstract}

36th International Cosmic Ray Conference - ICRC2019

24 July - 1 August, 2019

Madison, Wisconsin, USA

\footnotetext{
${ }^{*}$ Speaker.

${ }^{\dagger}$ for collaboration list see PoS(ICRC2019)1177
} 

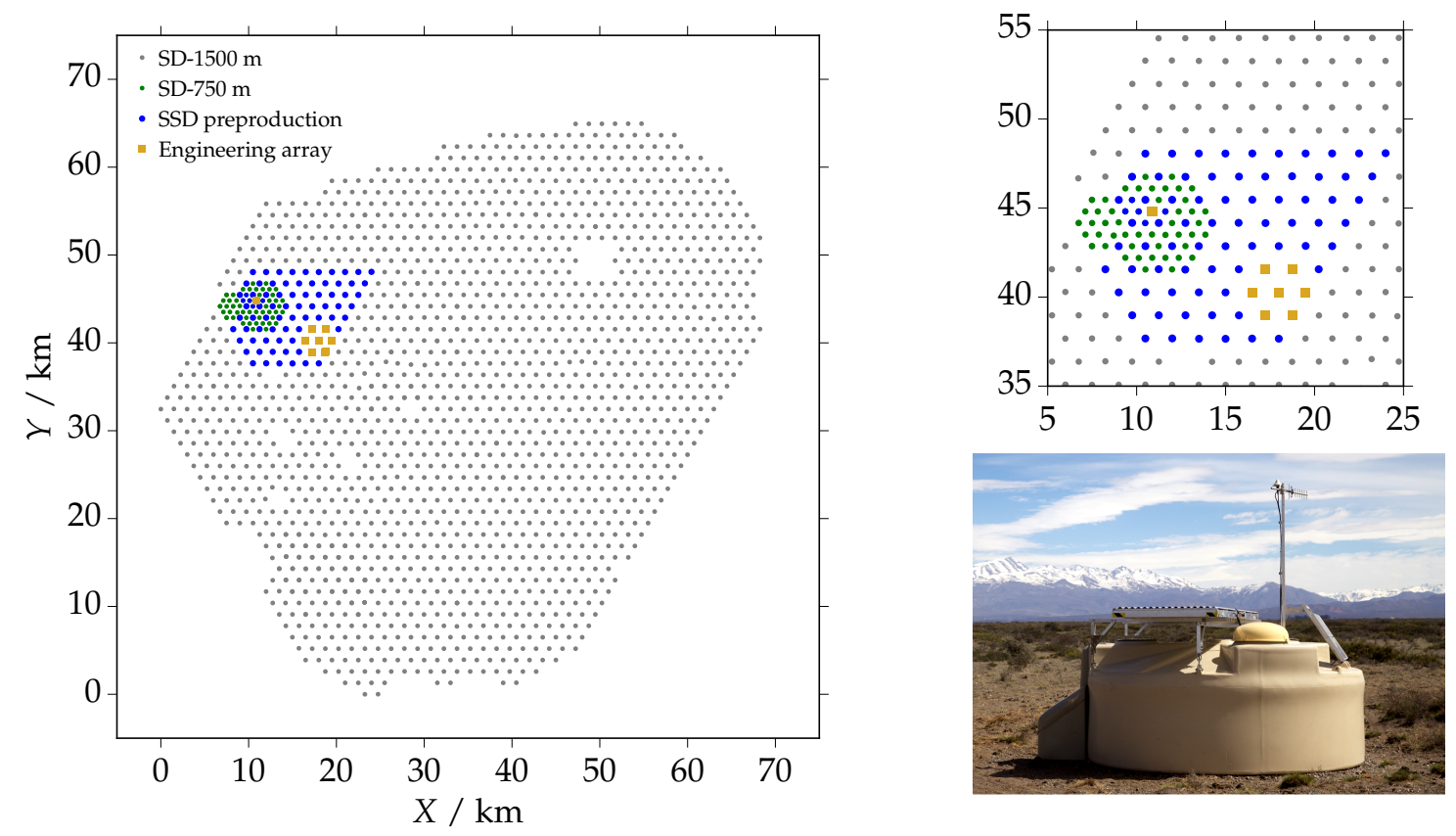

Figure 1: Left: Layout of the surface detector. Top right: Zoomed area containing the engineering array (golden squares) and SSD preproduction locations (blue dots). Bottom right: Photograph of an upgraded station of the surface detector.

\section{Introduction}

The main goal of the AugerPrime upgrade $[1,2]$ is to provide additional measurements of air showers which will allow for a better estimation of the mass composition of ultra-high energy cosmic rays. The key part of the upgrade is the installation of scintillator surface detectors on top of each of the existing water-Cherenkov detectors in the $3000 \mathrm{~km}^{2}$ surface detector (SD) array which can be seen in Fig. 1 (left). A complementary response of the two sub-detectors to shower particles allows us to disentangle the electromagnetic and the muonic shower components at the ground.

The SSD unit consists of an aluminum housing filled with 48 scintillator bars distributed between two modules covering a total area of $3.8 \mathrm{~m}^{2}$. Wavelength-shifting fibers guide photons produced in the scintillation process to a photomultiplier tube (PMT) located between the two modules. An additional element of AugerPrime is the upgrade of the SD electronics [3]. The upgraded board processes both of the SSD and WCD signals with higher sampling frequency (120 MHz instead of $40 \mathrm{MHz}$ ) and improved resolution in pulse amplitude (12 bits instead of 10 bits). Furthermore, an additional Small PMT (SPMT) in the WCD will increase the dynamic range of signals allowing measurements as close as $250 \mathrm{~m}$ from the shower core. A picture of an upgraded station can be seen in the bottom right of Fig. 1.

The first SSD units together with upgraded electronics and SPMT were deployed in the field in September of 2016. Twelve stations constitute the Engineering Array (EA) where nine are positioned using the standard $1500 \mathrm{~m}$ spacing and the remaining three form a multiplet at a $750 \mathrm{~m}$ spacing [4]. In March 2019, seventy-seven SSDs were connected to stations with non-upgraded 

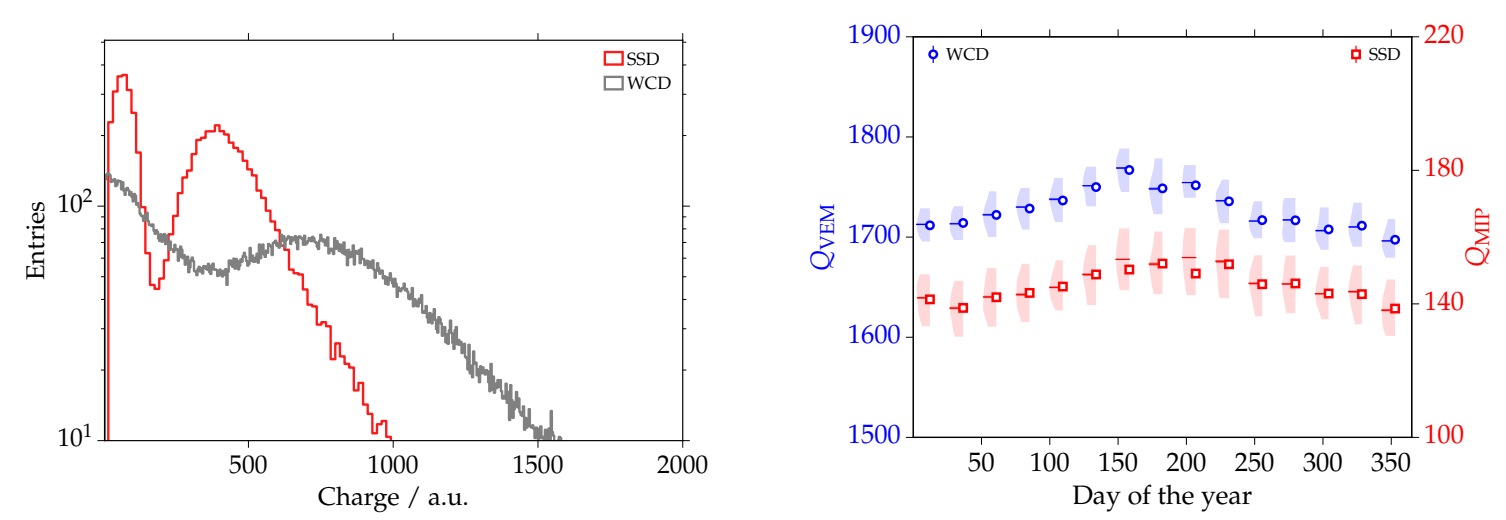

Figure 2: Left: Example of the "charge" distribution for the WCD (gray) and SSD (red) of an upgraded station at the engineering array. As the SSD is triggered by the WCD, the first peak of the charge distribution in the SSD is a pedestal (integral of baseline) in cases where the muon triggered the WCD but did not pass through the scintillator. Right: Profile of the variation with time of the VEM (blue circles) and MIP (red squares) charge. For each bin the spread of the distribution of $Q_{\mathrm{VEM}}$ and $Q_{\mathrm{MIP}}$ is shown by the blue and red shaded bands, respectively.
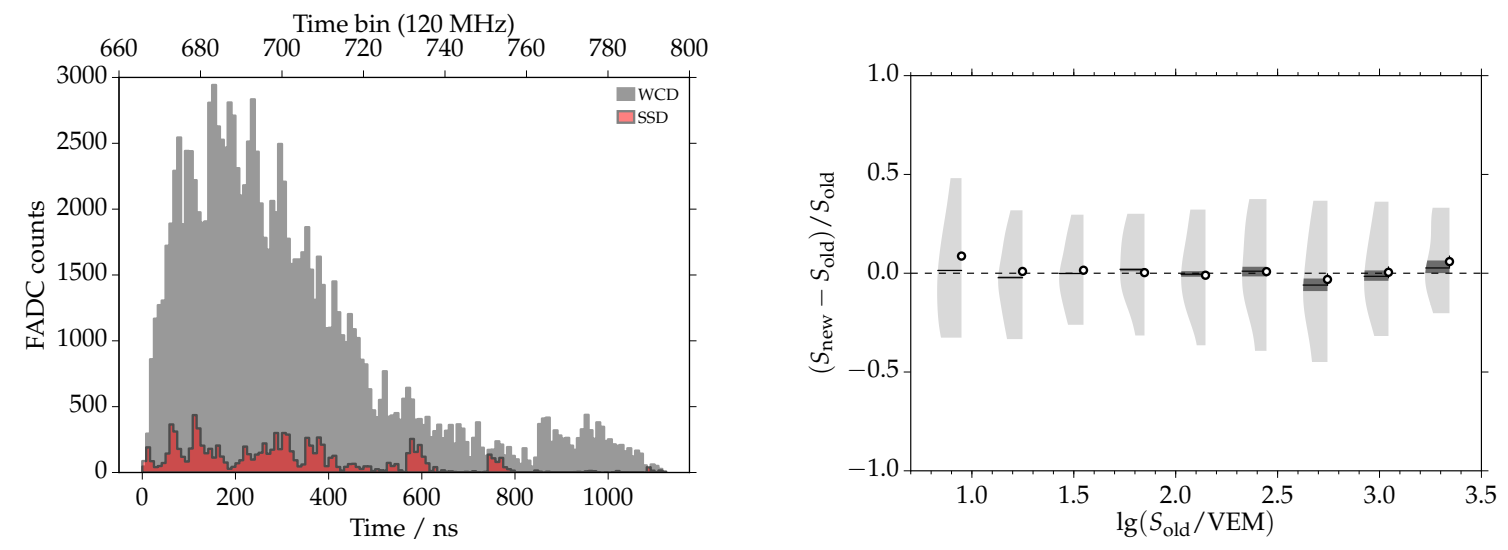

Figure 3: Left: Example of a time trace of an upgraded station located at $847 \mathrm{~m}$ from the shower core. The trace corresponds to one of the WCD PMTs (gray) and to the SSD (red). Right: Signal comparison from doublet measurements between old and new electronics as a function of the measured signal in a non-upgraded station. For this analysis 19337 doublet events with unsaturated signals were used.

electronics constituting the so-called SSD preproduction array. The area (of $\sim 120 \mathrm{~km}^{2}$ ) containing the engineering array and SSD preproduction locations can be seen in the top right of Fig. 1.

\section{Calibration and performance of the detectors}

The flux of particles that reach the ground is constantly measured and is used to provide a common reference for all signals recorded by the SD stations. Signals from the WCDs are expressed in units of VEM (vertical-equivalent muon), which corresponds to the average signal 
resulting from a vertical and centered muon traversing the tank, measured as the integrated PMT pulse over time, thus a "charge". An estimation of the VEM can be obtained from the spectrum of signals generated by atmospheric muons passing through the tank. An example of a "charge" distribution in a WCD can be seen in Fig. 2 (left). Such histograms exhibit two characteristic peaks. For the WCD, the first peak is due to small signals which are produced mainly by electromagnetic particles $\left(e^{ \pm}, \gamma\right)$ due to their low average energy deposited in water. The second peak is produced by atmospheric muons where the integrated signal is the result of a convolution of effects related to the muons' angular and energy spectra. By fitting the muon peak position, the value of the VEM charge $\left(Q_{\mathrm{VEM}}\right)$ is obtained [5]. Due to its dimensions, no direct calibration of the SPMT with muons is feasible. Selecting small shower events, the SPMT can be cross-calibrated with the large PMTs in such a way that the signal spectrum is correctly reconstructed up to about 20000 VEM. In the SSD muons and electrons deposit on average the same energy for a given traversed distance. The term MIP (minimum ionizing particle) is used to express the signals from the SSD. Analogous to the WCD, the average charge deposited by a MIP can be seen in the second peak of the charge histogram and the value of the MIP charge $\left(Q_{\text {MIP }}\right)$ can be inferred from the fit to that distribution. The evolution of the VEM and MIP charge with time can give us insight on the performance of the calibration. The violin plot $^{1}$ in Fig. 2 (right), shows the average behavior of the VEM and MIP charge for a station in the engineering array over one year of acquisition. The variation of $Q_{\mathrm{VEM}}$ and $Q_{\text {MIP }}$ related to seasonal modulations is apparent. At the same time, no aging effects seem to be present in the SSD.

When shower particles cross the detector, they produce time traces as a result of a convolution of the deposited energy with the response of the PMT. An example of a time trace can be seen in Fig. 3 (left). The calibrated signal $S$ is obtained from the integral of the trace and it is expressed in units of VEM and MIP for the WCD and SSD, respectively. A comparison of the WCD signals between stations with old and upgraded electronics is shown as a function of the signal in Fig. 3 (right). This comparison is performed using upgraded stations in the engineering array, which are part of a doublet with a non-upgraded station. A good agreement between the old and new electronics can be seen for a wide range of signals.

\section{Shower measurements with AugerPrime detectors}

Since the first SSD units were deployed in the engineering array, more than 28000 showers have been measured including both upgraded and standard stations in the event reconstruction. Due to its higher trigger rate, the majority of these showers were measured by EA stations at the SD-750 $\mathrm{m}$ array and with energies $\gtrsim 10^{17.5} \mathrm{eV}$. Fewer showers (but more energetic) have also triggered the upgraded stations of the SD-1500 m array. With the addition of the SSD units in the preproduction array, more than 150 showers at energies above $3 \mathrm{EeV}$ were measured including scintillator signals.

\subsection{Analysis of shower signals}

The SSD operates in slave mode to the WCD meaning that when a shower triggers the WCD,

\footnotetext{
${ }^{1}$ For each bin, the "violin" (shaded area) extends up to the $1 \sigma$ standard deviations of the distribution. The mean and median of the distribution are represented by markers and lines, respectively. The standard error on the mean is shown by the error bars while the darker area around the median line represents the $1 \sigma$ uncertainties on the median.
} 

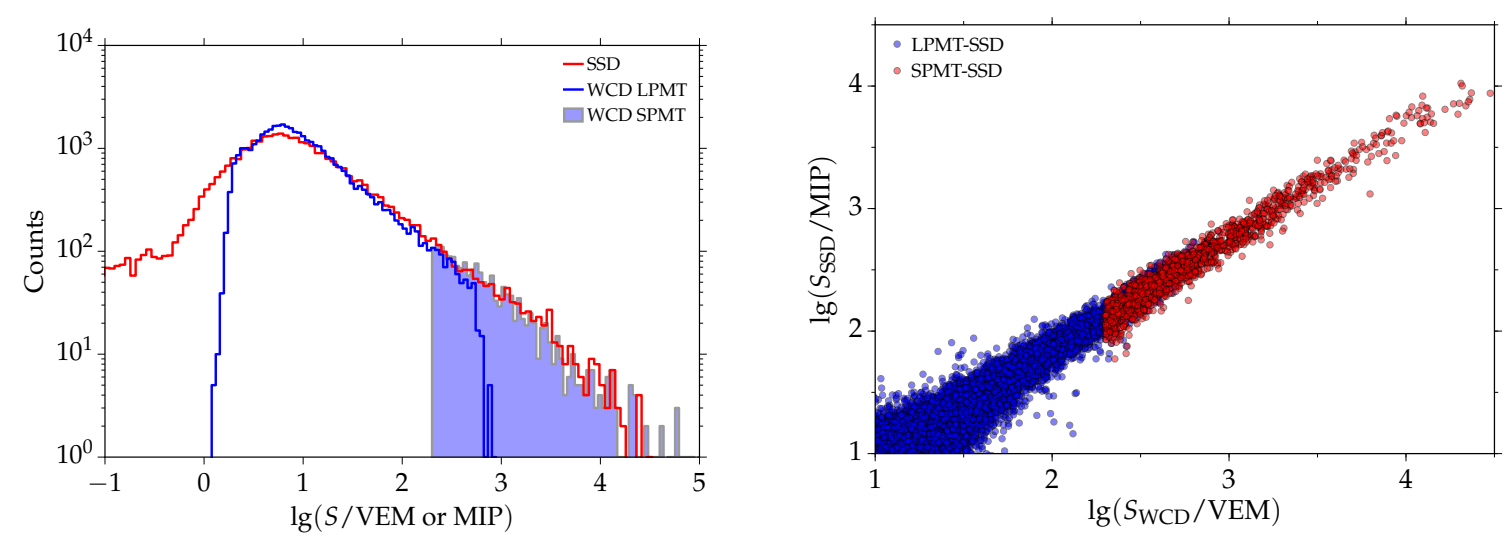

Figure 4: Left: Distributions of SSD (red) and WCD (blue) signals. The filled histogram shows signals from the SPMT. Right: Correlation between SSD and WCD signals using the large PMTs (blue) and the SPMT (red).
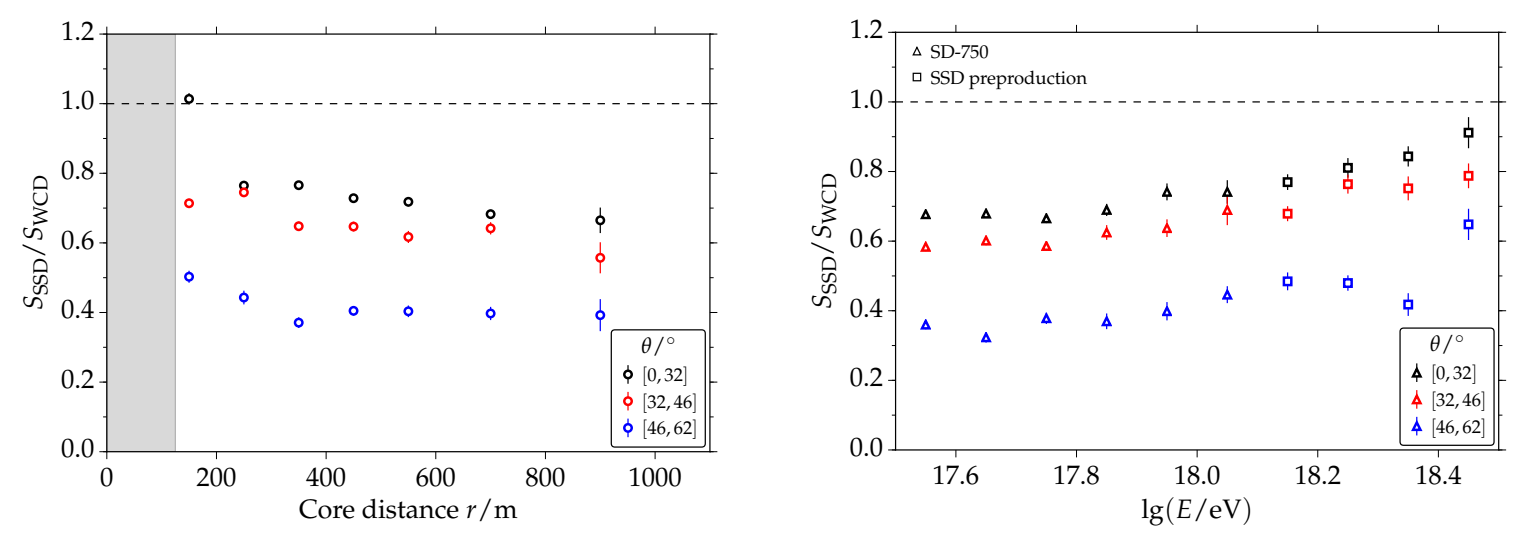

Figure 5: Left: Ratio of SSD and WCD signals as a function of the core distance, for different zenith angles of incidence. The gray area shows the region of saturated signals. Right: Ratio of signals as a function of the reconstructed energy. Data from the SD-750 m array (triangles) and SSD preproduction array (squares) are used. Ranges in zenith are chosen according to equal bins in $\sin ^{2} \theta$.

the information of the corresponding SSD is also read out. The signal distribution of both detectors is shown in Fig. 4 (left). The physics trigger is visible for the WCD with a sharp cut-off at low signals while for the SSD the signals can go down to baseline fluctuations in cases where no particles passed through the scintillator. The shape of the signal distribution in the SSD can be understood as a transition between the signal distribution due to baseline integration (no particle passing through) and/or small energy deposits in the scintillator (e.g., photons, corner-clipping particles, etc), and eventually the signal distribution produced by one or many particles traversing the detector. With the addition of the SPMT, the non saturated range of the WCD extends up to $\approx 20000$ VEM, as seen in Fig. 4 (right). The correlation between the signals measured by the SSD and the SPMT is also shown there.

The idea of using a scintillator detector in order to enhance the sensitivity of the surface de- 
tector to the mass of ultra-high energy cosmic rays relies on the differences in the SSD and WCD responses to shower components. While electromagnetic particles have smaller energy deposits than muons in water, both components deposit on average the same amount of energy in the scintillator. In this sense, the SSD is more sensitive to the electromagnetic component of the shower.

The ratio of SSD signals to WCD signals is shown in Fig. 5 (left) as a function of the distance to the shower core. The trend changes with the zenith angle. For vertical showers, the ratio $S_{\mathrm{SSD}} / S_{\mathrm{WCD}}$ is closer to 1 at distances closer to the shower core and decreases with increasing distance. For inclined showers, the ratio becomes nearly constant for distances $r>300 \mathrm{~m}$. In Fig. 5 (right) the same ratio is shown as a function of the reconstructed energy, using data from the EA stations located in the SD-750 m spacing array at energies below $10^{18.1} \mathrm{eV}$ and data from stations of the SSD preproduction array above that energy. The sensitivity of the SSD to the electromagnetic component, which has a faster increase with energy than muons, is shown by the up-going trend in this figure.

\subsection{Event reconstruction}

The surface detector records the footprint of the air shower at the ground. After passing the event selection criteria, standard shower reconstruction methods are applied to estimate the intrinsic properties of the primary cosmic ray. In the SD event reconstruction [6], the information about start times is used to reconstruct the arrival direction of the primary using a spherical model of the shower front.

After a first estimation of the arrival direction, a fit of the lateral distribution function (LDF) of signals at the ground is performed. This distribution arises from a convolution of the energy spectrum and the incoming direction of shower particles with the detector response. Different functional forms are used to describe signals at the ground which generally follow a power law with changing index. In this work, a modified Nishimura-Kamata-Greisen function is employed:

$$
S(r)=S\left(r_{\mathrm{opt}}\right)\left(\frac{r}{r_{\mathrm{opt}}}\right)^{\beta}\left(\frac{r+r_{\mathrm{s}}}{r_{\mathrm{opt}}+r_{\mathrm{s}}}\right)^{\beta+\gamma},
$$

where $\beta$ and $\gamma$ are the two slope parameters, and $r_{\text {opt }}$ and $r_{\mathrm{s}}$ are the optimal and scale distances, respectively. The optimal distance depends on the grid spacing and was determined to be $1000 \mathrm{~m}$ for the SD-1500 m array and $450 \mathrm{~m}$ for the SD-750 m array. The signal at the optimal distance is called the shower size $\left(S_{1000}, S_{450}\right)$ and is used to reconstruct the energy of the primary. The scale distance and the slope parameter $\gamma$ allow for more flexibility in the fit at distances far from the core. A change in the steepness of the LDF is expected due to the transition of the electromagnetic and the muonic lateral distributions, the latter being dominant at large distances as electromagnetic particles suffer from attenuation in the atmosphere.

A first example of an event reconstruction can be seen in Fig. 6 (left). The SPMT allows us to extend the unsaturated measure of the signal very close to the core of the shower. It can be seen how signals of the saturated stations can be corrected from $\approx 3000$ VEM to $\approx 7000$ VEM. The saturated and unsaturated traces of one of these stations are shown in Fig. 6 (right). Another example of an event measured with both the WCD and SSD is shown in Fig. 7 (left). The energy and arrival direction as well as the core position are estimated from the fit to the WCD signals. This information is then used for the fit of the SSD LDF. The profile of the average LDF for the WCD 

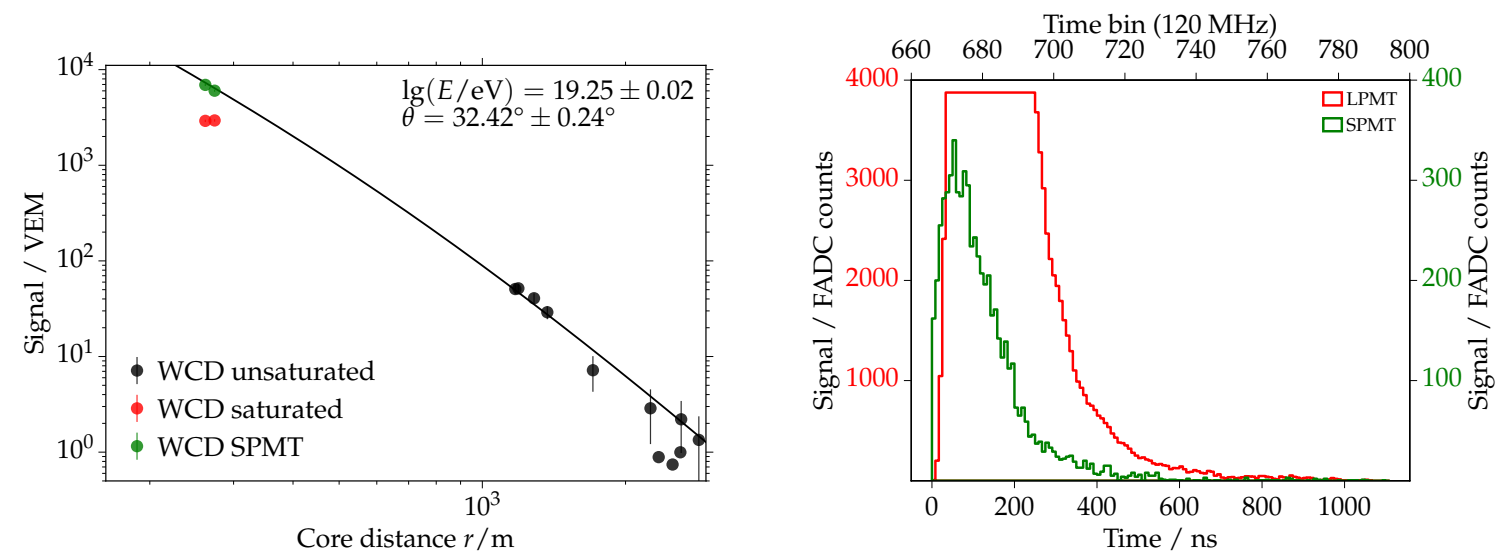

Figure 6: Left: Reconstruction of the event with ID: 46663172. The SPMT allows us to measure unsaturated signals close to the core improving the quality of the fit of the LDF. Right: Saturated (red) and unsaturated (green) traces of an upgraded station located at $260 \mathrm{~m}$ from the shower core.

and SSD signals can be seen in Fig. 7 (right). Each signal was normalized by the corresponding shower size to eliminate any degeneracy due to showers with different energies. As expected, the SSD has a steeper LDF than the WCD, with slightly larger signals close to the shower core and a faster fall off at large distances.
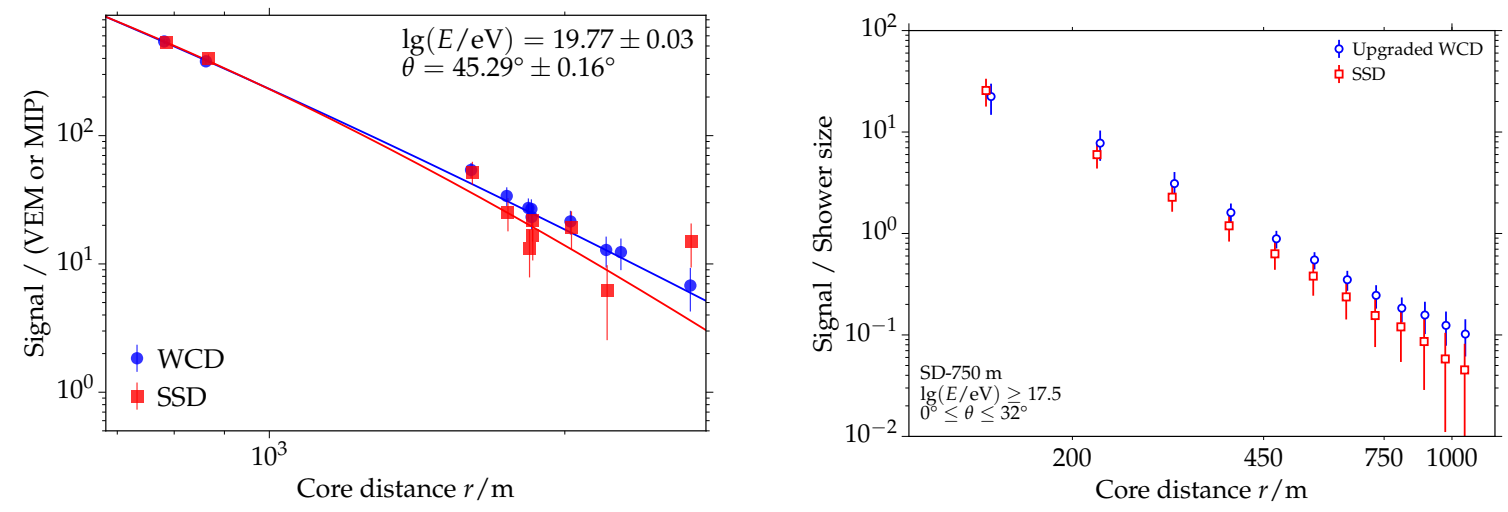

Figure 7: Left: Reconstruction of the event with ID: 53431866, with an energy of $\approx 60 \mathrm{EeV}$. The solid lines show the fit of the lateral distribution function of the WCD (blue) and the SSD (red) signals. Right: Profiles of the WCD and SSD signals (no saturation) normalized by the shower size. Reconstructed events of the SD-750 m array with energies $\geq 10^{17.5} \mathrm{eV}$ and zenith $\theta \in\left[0^{\circ}, 32^{\circ}\right]$ were used.

The contribution of the different components to the total signal is encoded in the time traces. The time structure is affected by the distance particles travel from their point of origin in the shower to the detector. In addition, both detectors have different time responses, for example, the signal in the WCD has a relatively larger spread due to the multiple reflections of Cherenkov light in the tank while in the SSD the time spread can be indicative of particles that were produced later in the shower development. An example of calibrated traces for the WCD and SSD can be seen in Fig. 8. 

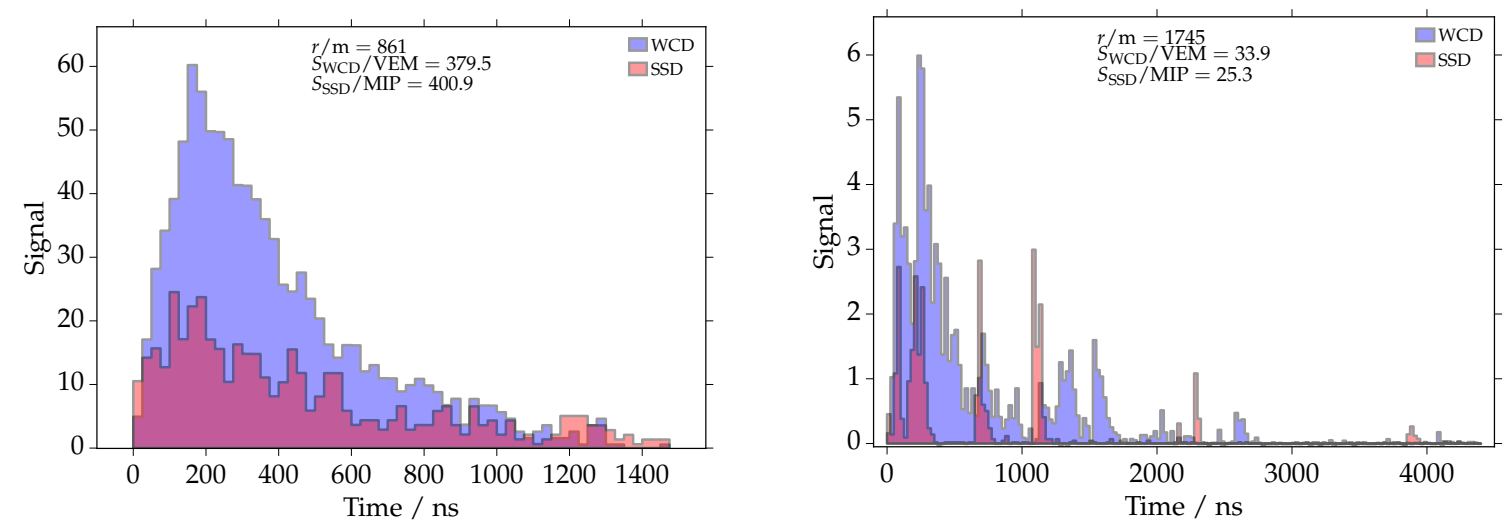

Figure 8: WCD and SSD traces from stations of the event shown in Fig. 7 (left), at different positions.

\section{Summary}

Stations of the AugerPrime upgrade have been taking data since they were deployed as part of the engineering array at the end of 2016. The results from the calibration have shown a good performance of the detectors as well as good agreement in the WCD signals comparing old and upgraded electronics. In addition, seventy-seven SSDs were deployed and started data acquisition in March 2019 increasing the wealth of events at higher energies. The dependency of the signal ratio with zenith, energy and distance, as well as the normalized LDFs, demonstrate the differences in the sensitivity of the two detectors to the different shower components. Furthermore, the additional SPMT extends the dynamic range of the WCD signals up to about 20000 VEM, allowing for a better reconstruction of events with stations close to the shower core.

\section{References}

[1] Pierre Auger Collaboration, The Pierre Auger Observatory Upgrade - Preliminary Design Report, arXiv:1604.03637

[2] A. Castellina for the Pierre Auger Collaboration, AugerPrime: the Pierre Auger Observatory Upgrade, proceedings of the Ultra High Energy Cosmic Rays 2018 Conference, EPJ Web Conf., 210, 06002 (2019).

[3] D. Nitz for the Pierre Auger Collaboration, these proceedings, PoS(ICRC2019)370.

[4] Z. Zong for the Pierre Auger Collaboration, proceedings of the 35th ICRC (Busan South Korea), PoS(ICRC2017)449.

[5] X. Bertou for the Pierre Auger Collaboration, Calibration of the surface array of the Pierre Auger Observatory, Nucl. Instrum. Meth. A 568, 839 (2006).

[6] D. Mockler for the Pierre Auger Collaboration, these proceedings, PoS(ICRC2019)353 